\title{
Fortalezas Familiares Y Consumo De Alcohol En Hombres Y Mujeres Adolescentes
}

\author{
Diana Betancourt Ocampo, Doctora \\ Alejandro González-González, Doctor \\ Universidad Anáhuac México, México \\ María Cristina Pérez Agüero, Doctora \\ Universidad Nacional Autónoma de México, México
}

doi: 10.19044/esj.2017.v13n26p281 URL:http://dx.doi.org/10.19044/esj.2017.v13n26p281

\begin{abstract}
The present study aimed to analyze the differences in family assets in adolescents, men and women, by level of alcohol consumption. For this, a non-probabilistic sample of 454 high school students was selected, with a mean age of 15.9 years old $(\mathrm{SD}=1.07)$; of which $49.1 \%$ were men and $50.9 \%$ were women. Three dimensions of the Parental Practice Scale (Andrade \& Betancourt, 2008) were used to assess family assets, which assesses the support and supervision of both parents; in addition, the scale house rules of Sánchez-Xicotencatl (2015) was used. To measure alcohol consumption, four indicators were used to evaluate the behavior's presence, the age of onset, as well as frequency and quantity (Andrade \& Betancourt, 2008). The results showed that $57 \%$ of men and $53.8 \%$ of women reported that they had consumed alcohol. Regarding the differences in family assets by the categories of the alcohol consumption indicator, the findings showed significant differences house rules (in both men and women), and in the men's case differences were found in maternal supervision and in women, differences were found in the support-supervision of the father. The results allow us to have elements of the role of the assets evaluated in the alcohol consumption in adolescent population, as well as the differential effect by the sex of the adolescents.
\end{abstract}

Keywords: Adolescent, Alcohol consumption, Parents, Rules, Assets

\section{Resumen}

El presente artículo de investigación tuvo como propósito analizar las diferencias en las fortalezas familiares en adolescentes, hombres y mujeres, por nivel de consumo de alcohol. Para lo cual se seleccionó una muestra no probabilística de 454 estudiantes de nivel bachillerato, con un promedio de 
edad de 15.9 años (DE=1.07); de los cuales, $49.1 \%$ fueron hombres y $50.9 \%$ mujeres. Para evaluar las fortalezas familiares, se utilizaron tres dimensiones de la Escala de Prácticas Parentales (Andrade \& Betancourt, 2008), las cuales evalúan el apoyo y la supervisión de ambos padres; además, se utilizó la escala de Reglas en casa de Sánchez-Xicotencatl (2015). Para medir el consumo de alcohol se utilizaron cuatro indicadores que evalúan la presencia de la conducta, la edad de inicio, así como la frecuencia y cantidad (Andrade \& Betancourt, 2008). Los resultados mostraron que 57\% de los hombres y $53.8 \%$ de las mujeres reportaron que si han consumido alcohol alguna vez en su vida. Respecto a las diferencias en las fortalezas familiares por las categorías del indicador de consumo de alcohol, los hallazgos mostraron diferencias significativas en las reglas en casa (tanto en hombres como en mujeres), además en el caso de los hombres se encontraron diferencias en la supervisión materna y en las mujeres, se encontraron diferencias en el apoyosupervisión del padre. Los resultados permiten contar con elementos del papel que tienen las fortalezas evaluadas en el consumo de alcohol en población adolescente, así como del efecto diferencial por el sexo de los adolescentes.

Palabras-claves: Adolescentes, Consumo de Alcohol, Padres, Reglas, Fortalezas

\section{Introducción}

De acuerdo con algunos autores la adolescencia es un período en el que incrementan los conflictos con los padres, así como la inestabilidad emocional y las conductas de riesgo (Arnett, 1999; Larson, \& Richards, 1994; Laursen, Coy, \& Collins, 1998; Steinberg, \& Morris, 2001), sin embargo, hoy en día existe evidencia que sugiere que esta imagen caótica de la adolescencia no puede generalizarse. De hecho, es a finales del siglo XX que surge el enfoque del desarrollo positivo de los jóvenes (Positive Youth Development, PYD, por sus siglas en inglés), el cual destaca las potencialidades de los jóvenes más que sus supuestas incapacidades (Damon, 2004).

Algunos autores (Benson, Mannes, Pittman \& Ferber, 2004; Lerner et al., 2005) sugieren que el PYD surge como un paradigma alternativo a la orientación tradicional del déficit, ya que esta perspectiva busca identificar factores particularmente importantes para predecir resultados favorables tanto físicos, como emocionales y sociales en los jóvenes. Heck y Subramaniam (2009) realizaron una revisión de los modelos que más se han utilizado en la PYD, los autores reportan que uno de los modelos que cuenta con mayor respaldo empírico es el de las fortalezas del desarrollo, el cual fue propuesto por investigadores del Search Institute of Minnesota, el cual 
considera que las fortalezas son un conjunto de "bloques de construcción" (Leffert et al., 1998, p.209) que, cuando están presentes ayudarán a mejorar los resultados importantes del desarrollo, concebidos como la reducción de conductas que comprometan la salud de los jóvenes y el incremento de su desempeño académico y otros indicadores de resultados positivos.

De acuerdo con este modelo, hay 40 fortalezas del desarrollo que se agrupan en dos grandes categorías: internas (20) y externas (20); las primeras, se definen como aquellas que se desarrollan gradualmente a lo largo del tiempo (por ejemplo, responsabilidad, visión positiva al futuro, honestidad, motivación al logro, etc.). Por otro lado, las fortalezas externas se refieren a las oportunidades de desarrollo positivo que se dan en la interacción adulto-adolescente-pares y que son reforzadas por instituciones (por ejemplo, apoyo y comunicación familiar, límites familiares, influencia positiva de pares, etc.) (Benson, 2002, 2007; Leffert et al., 1998; Scales, 1999).

Por otro lado, son diversos los estudios realizados desde la PYD que evalúan algunas conductas de riesgo asumiendo que aquellos jóvenes que tengan un mejor desarrollo positivo evitarán el involucramiento en este tipo de comportamientos (Evans et al., 2004; Murphy, Lamonda, Carney, \& Duncan, 2004; Oman et al., 2004; Oman, Vesely, \& Aspy, 2005). Dentro de las conductas de riesgo, el consumo de alcohol es considerado en México como un problema de salud pública, esto debido a que los adolescentes inician su consumo cada vez a edades más tempranas y ha incrementado el consumo en mujeres. De acuerdo con los datos de la Encuesta de Estudiantes de México (Villatoro et al., 2016), en nuestro país $74.5 \%$ de los estudiantes varones de nivel bachillerato reportaron que alguna vez en la vida han consumido bebidas alcohólicas, en el caso de las mujeres de este mismo nivel escolar el porcentaje fue de $73.3 \%$; respecto al consumo de alcohol en el último año, los resultados mostraron porcentajes que fue ligeramente mayor el porcentaje en los hombres $(56.5 \%)$ que de las mujeres $(52.1 \%)$.

Respecto al papel que juegan algunas de las fortalezas externas sobre el consumo de alcohol en población adolescente, existe evidencia que sugiere que las conductas parentales como la supervisión se asocia fuertemente con el consumo de sustancias en adolescentes, por ejemplo Stavrinides, Georgiou y Demetriov (2010) realizaron un estudio donde analizaron el efecto de dos recursos de supervisión parental sobre las actividades de los adolescentes (tanto el conocimiento de los padres sobre las actividades que realizan los jóvenes como la comunicación de los propios adolescentes a sus padres acerca de sus actividades) sobre conductas problemáticas, los autores encontraron relaciones significativas entre la supervisión parental y el consumo de alcohol, sin embargo, fue más 
importante la relación con la dimensión de la información que proporciona el joven sobre sus actividades que con la del conocimiento de los padres.

En este mismo sentido, Tebes et al., (2011) reportan que altos niveles de supervisión parental pueden reducir la probabilidad de iniciación del consumo de alcohol en población adolescente, sin embargo, ya que los adolescentes han iniciado su consumo la supervisión parental no tiene un efecto significativo en la frecuencia con la que consumen. Becerra, Castillo, Ayón y Blanchard (2014) encontraron datos que apoyan los estudios anteriores, ya que reportan un efecto significativo de la supervisión parental sobre el consumo de alcohol y tabaco, no obstante, los autores sugieren que a pesar de que la supervisión de los padres es relevante en el consumo de sustancias, la influencia de los pares parece tener una mayor influencia. Por otro lado, Pagan y DePaulo (2013) realizaron un estudio donde analizaron el rol mediador del apoyo y la supervisión parental en el consumo de alcohol de estudiantes México Americanos, los autores encontraron que los adolescentes varones presentaron un mayor riesgo de consumir alcohol en contraste con las mujeres, esto sin importar si percibían altos o bajos puntajes de apoyo y supervisión parental, lo cual es un dato interesante, ya que de acuerdo a estos resultados el sexo de los jóvenes pareciera ser un factor que pesa más que algunas de las conductas parentales.

Por otra parte, Habib et al., (2010) exploraron las interacciones entre la relación del adolescente con el padre (apego) y las reglas dentro del hogar con el consumo de tres indicadores de consumo de alcohol: consumo alguna vez, consumo actual y emborrachamiento; los investigadores encontraron que el apego fue variable predictora solo para el consumo de alguna vez en la vida y de emborrachamiento, no obstante, las reglas dentro del hogar fueron variables significativas en los tres indicadores del consumo de alcohol.

Marshal y Chassin (2000) encontraron que tanto el apoyo como la disciplina parental se asocian con la frecuencia del consumo de alcohol en una muestra de adolescentes, asimismo, dichos autores sugieren que el apoyo parental si bien no es una variable que consistentemente se asocie de manera directa con el consumo de sustancias, sí se considera una variable que explica la relación con pares consumidores la cual se ha reportado consistentemente en la literatura como predictora del consumo de sustancias en adolescentes.

Finalmente, cabe mencionar la revisión de la literatura que realizaron Ryan, Jorm y Lubman (2010) para identificar qué estrategias parentales se asocian con el consumo de alcohol en población adolescente. Dentro de las diferentes estrategias que reportan los autores, un mayor número de estudios han apoyado que la supervisión parental tiene un efecto significativo tanto para la edad de inicio (10 estudios) como para altos niveles de consumo de alcohol (14 estudios); en este mismo sentido, las reglas y disciplina dentro 
del hogar, también mostraron efectos significativos para el inicio y para el consumo elevado, no obstante el número de estudios reportados fue menor (solo diez estudios). Por lo que se refiere al apoyo parental, los resultados indicaron que de los tres estudios que se analizaron con respecto a la edad de inicio del consumo, no mostraron efectos significativos, pero para el consumo alto los 14 estudios analizados si tuvieron un impacto significativo.

Como se puede apreciar, la mayoría de la literatura ha sido consistente en el rol que juega el apoyo y la supervisión parental, así como las reglas dentro del hogar en el consumo de alcohol en población joven y a pesar de que los datos epidemiológicos indican niveles distintos de consumo entre hombres y mujeres, no todos las investigaciones consideran las diferencias por sexo, es por ello, y partiendo desde el PYD que el objetivo del presente estudio fue analizar las diferencias en algunas de las fortalezas externas, específicamente en fortalezas familiares, en hombres y mujeres adolescentes por nivel de consumo de alcohol.

\section{Método}

\section{Participantes}

Se seleccionó una muestra no probabilística de 454 adolescentes, estudiantes de nivel bachillerato, de los cuales $49.1 \%$ fueron hombres y $50.9 \%$ mujeres, con un promedio de edad de 15.9 años (DE=1.07). E1 30.4\% eran estudiantes de escuelas públicas y $69.6 \%$ de escuelas privadas. El $63.1 \%$ de los adolescentes informaron que vivían en familias biparentales y $36.9 \%$ reportaron vivir con uno solo de los padres.

\section{Instrumentos}

Las fortalezas que se evaluaron fueron: apoyo de la madre ( $\square=.93)$, supervisión de la madre ( $\square=.79$ ), apoyo y supervisión del padre ( $\square=.96$ ) y reglas en la casa $(\square=.71)$, todas fueron escalas tipo Likert con cinco opciones de respuesta (de Nunca a Siempre). Las primeras tres, corresponden a dimensiones de la Escala de Prácticas Parentales de Andrade y Betancourt (2008a); la escala de reglas en casa fue tomada del instrumento de SánchezXicotencatl (2015) quien adaptó para población mexicana el Cuestionario de Salud y Desarrollo de los Adolescentes (Adolescent Health and Development Questionnaire). Por lo que respecta al consumo de alcohol, se utilizaron cuatro indicadores que evalúan la presencia de la conducta, la edad de inicio, así como la frecuencia y cantidad (Andrade \& Betancourt, 2008b).

\section{Procedimiento}

Se acudió con las autoridades escolares para explicarles el objetivo del estudio y solicitar su permiso para aplicar los instrumentos dentro de las instalaciones de la escuela, las autoridades escolares se encargaron de contar 
con la aprobación de los padres para la aplicación de los instrumentos con los estudiantes. Los instrumentos fueron auto-aplicables y los alumnos los respondieron de forma grupal dentro de los salones de clase, antes de que respondieran, se les explicó el propósito del estudio y se les solicitó su participación voluntaria, se les garantizó la confidencialidad de la información y se respondieron dudas de aquellos que así lo solicitaron.

\section{Resultados}

Para determinar la distribución de los adolescentes en cada uno de los indicadores de consumo de alcohol, se realizaron análisis de frecuencias por separado para hombres y para mujeres. Respecto al consumo alguna vez en la vida de alguna bebida alcohólica, el $57 \%$ de los hombres reportaron que sí han consumido y en el caso de las mujeres fue el 53.8\%. En cuanto a la edad del primer consumo, no se encontraron diferencias estadísticamente significativas $[t(1,305)=-1.13, p>.05]$ entre hombres $(M=13.64, D E=1.80)$ y mujeres $(M=13.85, D E=1.44)$.

Se diseñó un indicador con la frecuencia del consumo de alcohol, el cual incluye desde los jóvenes que nunca han consumido esta sustancia hasta aquellos que la han consumido más de dos ocasiones en el mes previo a la recolección de datos, en la Figura 1 se presentan los resultados en cada una de las categorías de consumo por sexo.

Figura 1

Distribución de los participantes por nivel de consumo de alcohol por sexo.

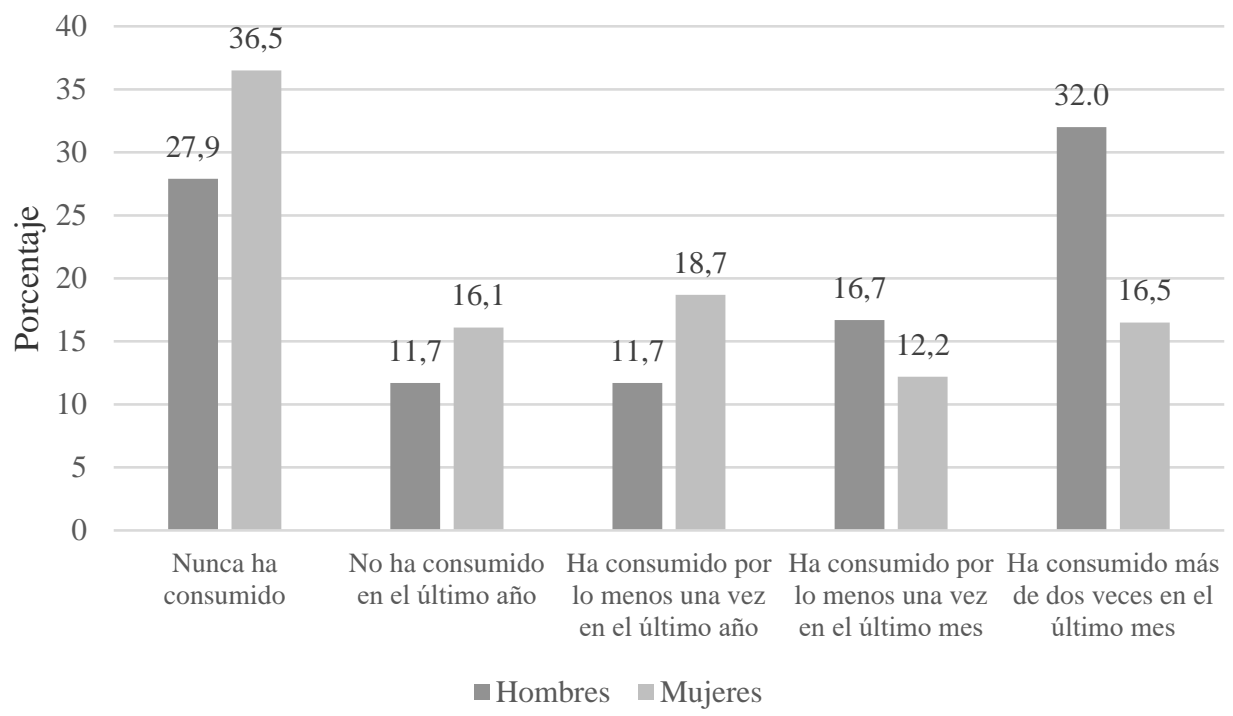

Como se puede observar, se encontró una relación estadísticamente significativa entre el indicador del consumo de alcohol y el sexo de los participantes ( $\left.X^{2}=20.56, p<.001\right)$, donde fue mayor el porcentaje de mujeres 
que informaron que no han iniciado su consumo de alcohol en contraste con los varones, lo cual se mantiene relativamente constante en las categorías de aquellos que no han consumido en el último año o lo han hecho una sola ocasión. Sin embargo, en las categorías del consumo en el último mes (de una ocasión o más), fue mayor la proporción de hombres que de mujeres (Figura 1).

Para determinar las diferencias en las fortalezas evaluadas por categoría de consumo se realizaron análisis de varianza de una vía por hombres y mujeres. En cuanto a los resultados de los hombres, se encontraron diferencias estadísticamente significativas en la supervisión materna y en la dimensión de reglas en la casa, donde las pruebas post hoc indicaron que en el caso de la supervisión materna las diferencias significativas $(p<.05)$ fueron entre los adolescentes que alguna vez han consumido alcohol pero no en el último año y los estudiantes que han consumido más de dos ocasiones en el último mes, donde el primer grupo reportó que percibe una mayor supervisión por su mamá que los que han consumido en un mayor número de ocasiones en el mes previo a la recolección de datos (ver Tabla 1). Por lo que se refiere a las reglas en casa, las pruebas de comparaciones múltiples mostraron que las diferencias fueron entre los estudiantes que han consumido al menos en una ocasión en el último mes y los que han consumido de dos a más veces en el último mes, donde el primer grupo percibe que con mayor frecuencia se establecen reglas dentro de su hogar en contraste con los que presentan un mayor consumo.

Tabla 1 Diferencias en las fortalezas externas por nivel de consumo en hombres.

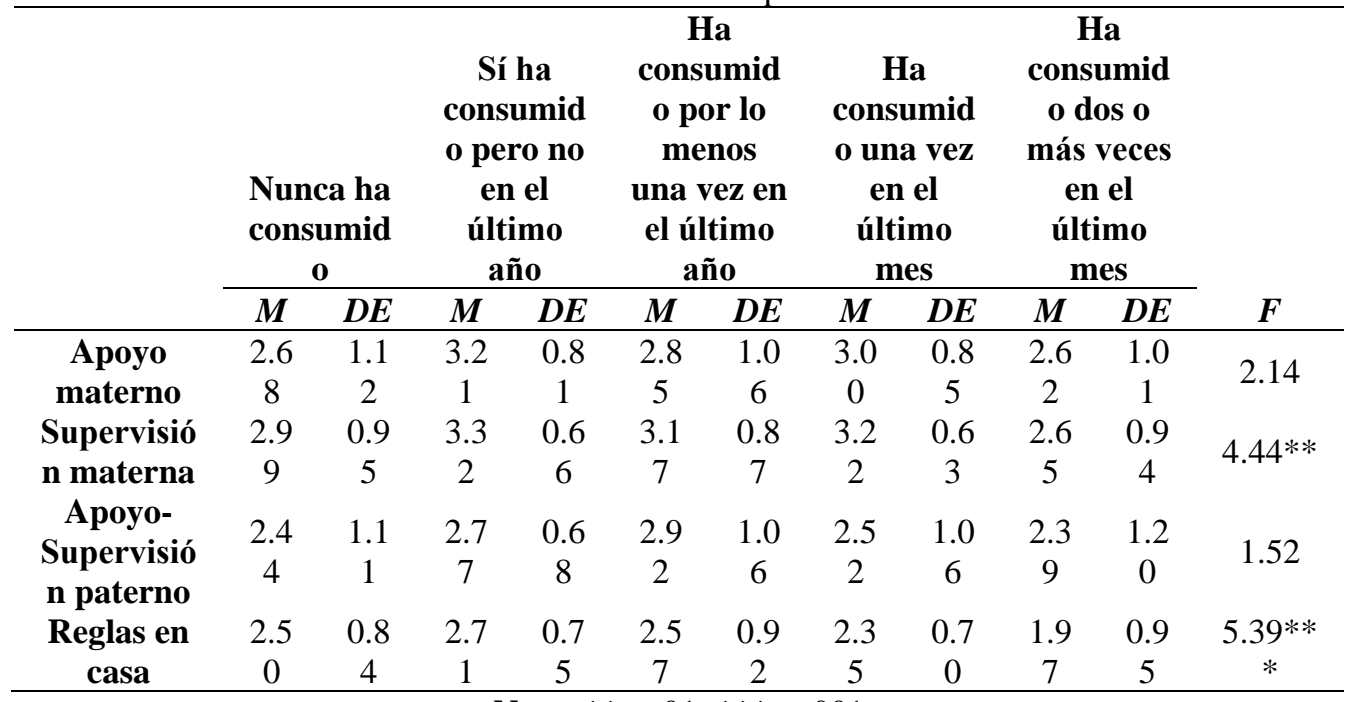

Nota. $* * p<.01, * * * p<.001$ 
En cuanto a los resultados de las mujeres (Tabla 2), estos mostraron diferencias significativas en las dimensiones de apoyo-supervisión paterna y las reglas en casa, donde en el caso de la supervisión del papá las pruebas post hoc indicaron diferencias significativas $(p<.05)$ en las adolescentes que nunca han consumido alcohol o que no lo han hecho en el último año respecto a aquellas que han consumido de dos o más ocasiones en el último mes, donde las primeras fueron quienes percibieron una mayor supervisión por parte de su papá que las que tienen consumo en el último mes. En la dimensión de reglas en la casa, las estudiantes que no han consumido o no lo han realizado en el último año percibieron significativamente $(p<.05)$ más reglas dentro de su hogar que las adolescentes que han consumido alcohol en el último mes.

Tabla 2 Diferencias en las fortalezas externas por nivel de consumo en mujeres.

\begin{tabular}{|c|c|c|c|c|c|c|c|c|c|c|c|}
\hline & \multicolumn{2}{|c|}{$\begin{array}{l}\text { Nunca ha } \\
\text { consumido }\end{array}$} & \multicolumn{2}{|c|}{$\begin{array}{c}\text { Sí ha } \\
\text { consumido } \\
\text { pero no en } \\
\text { el último } \\
\text { año } \\
\end{array}$} & \multicolumn{2}{|c|}{$\begin{array}{c}\text { Ha } \\
\text { consumido } \\
\text { por lo } \\
\text { menos una } \\
\text { vez en el } \\
\text { último año }\end{array}$} & \multicolumn{2}{|c|}{$\begin{array}{c}\text { Ha } \\
\text { consumido } \\
\text { una vez en } \\
\text { el último } \\
\text { mes } \\
\end{array}$} & \multicolumn{2}{|c|}{$\begin{array}{c}\text { Ha } \\
\text { consumido } \\
\text { dos o más } \\
\text { veces en el } \\
\text { último } \\
\text { mes } \\
\end{array}$} & \multirow[b]{2}{*}{$F$} \\
\hline & $M$ & $D E$ & $M$ & $D E$ & $M$ & $D E$ & $M$ & $D E$ & $M$ & $D E$ & \\
\hline $\begin{array}{c}\text { Apoyo } \\
\text { materno }\end{array}$ & 3.12 & 1.00 & 3.03 & 0.88 & 2.95 & 1.03 & 2.63 & 1.16 & 2.73 & 1.14 & 1.70 \\
\hline $\begin{array}{c}\text { Supervisión } \\
\text { materna } \\
\text { Apoyo- }\end{array}$ & 3.28 & 0.87 & 3.31 & 0.89 & 3.22 & 0.81 & 3.00 & 1.00 & 2.88 & 0.90 & 1.90 \\
\hline $\begin{array}{c}\text { Supervisión } \\
\text { paterno }\end{array}$ & 2.35 & 1.27 & 2.32 & 0.95 & 2.28 & 1.34 & 2.51 & 1.10 & 1.67 & 1.17 & $2.66^{*}$ \\
\hline $\begin{array}{c}\text { Reglas en } \\
\text { casa }\end{array}$ & 2.56 & 0.96 & 2.58 & 0.81 & 2.45 & 0.96 & 2.07 & 0.86 & 2.08 & 0.82 & $3.08 *$ \\
\hline
\end{tabular}

Nota. $* p<.05$

\section{Discusión}

De acuerdo con los datos epidemiológicos sobre el consumo de alcohol (Villatoro et al., 2016), en México, aproximadamente tres cuartas partes de los estudiantes de nivel bachillerato han consumido alguna vez en su vida bebidas alcohólicas, estos datos son mayores a lo encontrado en la presente investigación donde la proporción de jóvenes que informó que ha consumido alcohol es menor; no obstante, algo que concuerda con los datos a nivel nacional es que sigue siendo ligeramente mayor la proporción de varones que consumen que de mujeres, proporción que en los últimos años se ha ido aminorando y que hoy en día representa una de las preocupaciones para el sector salud de nuestro país (Medina-Mora et al., 2012), es por ello, que es primordial contar con elementos que puedan favorecer el decremento del cosumo de alcohol, así como el retraso del inicio del consumo, sobretodo 
en población joven, lo cual sería recomendable considerara estas diferencias por sexo, tanto para el diagnóstico como en las intervenciones.

Por otro lado, de acuerdo con la literatura una de las fortalezas externas que se ha asociado con el no consumo de alcohol es la supervisión de los padres, donde en general los estudios han mostrado que aquellos adolescentes que perciben que sus padres tienen una mayor supervisión o monitoreo de sus actividades son los que tienden a no consumir o a retrasar su inicio de consumo de sustancias (Becerra et al., 2014; Tebes et al., 2011; Ryan et al., 2010; Stavrinides et al., 2010), lo cual fue corroborado en el presente estudio, donde los hallazgos indicaron efectos diferenciales, tanto por el sexo de los padres como por el sexo de los hijos, donde en el caso de los varones, aquellos que han consumido alcohol pero no lo han realizado en el año previo al estudio percibieron una mayor supervisión materna que quienes han consumido más de dos ocasiones durante el último mes, lo cual es ligeramente parecido en el grupo de mujeres, sólo que en ese caso solo se encontraron diferencias significativas en la supervisión del padre. Es decir, que en el caso de los varones de la muestra estudiada, la supervisión de la madre fue la que marco diferencias por categoría de consumo y para las mujeres, fue la supervisión del padre. Los hallazgos del efecto diferencial del sexo de los padres y de los hijos, ha sido poco considerado en los estudios previos, ya que la gran mayoría de éstos evalúan la supervisión de ambos padres y son pocos los estudios donde las conductas de los padres se valoran por separado. Es por lo anterior, que se recomienda para futuros estudios tener en consideración que la relación que se establece entre padres e hijos es diferente de acuerdo a sexo del adolescente y del padre.

Habib et al., (2010), Marshal y Chassin (2000) y Ryan et al., (2010) reportaron que las reglas y disciplina dentro del hogar se asocian consistentemente con el no consumo de sustancias, lo cual fue corroborado con los resultados del presente estudio, ya que tanto en la muestra de varones como de mujeres, se encontraron efectos significativos, sin embargo, en el caso de las adolescentes las diferencias fueron entre los grupos de menos consumo (nunca ha consumido y si ha consumido pero no en el último año) con las que consumieron en el mes previo al estudio y para el caso de los varones, las diferencias se encontraron con el grupo que ha consumido una vez en el último mes y los que han consumido más de una ocasión, donde tanto en hombres como en mujeres, los adolescentes que consumen alcohol con menor frecuencia son quienes perciben más reglas dentro de casa. Como se puede apreciar, las reglas dentro del hogar representan una fortaleza para que los adolescentes presenten una baja frecuencia en el consumo de alcohol, no obstante, en concordancia con lo mencionado previamente, el sexo de los adolescentes fue un factor que marcó diferencias y esto podría deberse a aspectos culturales ya que regularmente a los varones se les dan un poco más 
de libertades que a las mujeres, por lo tanto el aspecto cultural debería considerarse en el desarrollo de programas de prevención de consumo de sustancias.

En cuanto al apoyo parental, los resultados mostraron que éste no tuvo un efecto importante en la frecuencia de consumo de los adolescentes, en especial en las mujeres, ya que ni el apoyo de la madre ni del padre mostraron diferencias significativas. En el caso de los hombres, los resultados mostraron que para el apoyo materno no se encontraron diferencias significativas por frecuencia de consumo, no obstante, para el caso del padre sí se encontraron diferencias significativas, esto tal vez se debe, a que de acuerdo a la estructura del instrumento el apoyo del padre está en la misma dimensión de la supervisión, así que no se tendría la certeza que las diferencias fueran en una sola de las áreas evaluadas. Estos hallazgos no concuerdan con previos estudios (Habib et al., 2010; Marshal \& Chassin, 2000; Ryan et al., 2010) en cuanto al rol de la percepción del apoyo parental sobre el consumo de alcohol, no obstante, la misma literatura ha sido clara en mencionar que pareciera que el apoyo no tiene un efecto directo sobre el consumo si no que pareciera ser una variable mediadora de otros factores como el involucramiento con pares que consumen (Marshal \& Chassin, 2000), de ahí, que se recomiende realizar más investigación al respecto, para contar con evidencia contundente del papel del apoyo parental sobre el consumo de sustancias en adolescentes.

A manera de conclusión, se puede mencionar que el presente estudio aportó elementos que permiten comprender de manera más puntual, como la supervisión y la percepción de reglas dentro de casa pueden considerarse como fortalezas externas de los adolescentes para el bajo consumo de alcohol. En consonancia con la PYD, los resultados de este estudio permiten comprender a la adolescencia como un período que si bien, regularmente se le ha asociado con el inicio de conductas que pueden afectar su bienestar (entre ellas el consumo de sustancias), es más que ello, es un periodo donde pueden promoverse una serie de fortalezas o recursos que pueden contribuir en su desarrollo saludable y que la salud, va más allá de la ausencia de conductas de riesgo.

\section{References:}

1. Andrade, P. P., \& Betancourt, O. D. (2008a). Prácticas parentales: Una medición integral (pp. 561-565). En: Rivera, A. S., Díaz-Loving, R., Sánchez, A. R., y Reyes, L. I. (Eds.) La Psicología Social en México XII, México: AMEPSO.

2. Andrade, P.P., \& Betancourt, O.D. (2008b). Factores individuales, familiares y sociales y conductas de riesgo en adolescentes (pp. 181- 
213). En: Andrade, P.P., Cañas, M. J.L. y Betancourt, O.D. (comp.) Investigaciones psicosociales en adolescentes. México: UNICACH.

3. Arnett, J. J. (1999). Adolescent storm and stress, reconsidered. American Psychologist, 54(5), 317-326. doi: http://dx.doi.org/10.1037/0003-066X.54.5.317

4. Becerra, D., Castillo, J. T., Ayón, C., \& Blanchard, K. N. (2014). The moderating role of parental monitoring on the influence of peer prodrug norms on alcohol and cigarette use among adolescents in Mexico. Journal of Child \& Adolescent Substance Abuse, 23(5), 297306. doi: http://dx.doi.org/10.1080/1067828X.2013.869138

5. Benson, P. L. (2002). Adolescent development in social and community context: A program of research. New Directions for Student Leadership, 95, 123-147. doi: 10.1002/yd.19.

6. Benson, P. L. (2007). Developmental assets: an overview of theory, research and practice (pp. 1-58). En: Rainer K. Silbereisen \& Richard M. Lerner (Eds.), Approaches to Positive Youth Development. Los Angeles: Sage Publications.

7. Benson, P., Mannes, M., Pittman, K., \& Feber, T. (2004). Youth development, developmental assets, and public policy. En: R. M. Lerner \& L. Steinberg (Eds.), Handbook of Adolescent Psychology (pp. 781-814). New York, NY: Wiley.

8. Damon, W. (2004). What is positive youth development? The Annals of the American Academy of Political and Social Science, 591, 13-24.

9. Evans, A. E., Sanderson, M., Griffin, S. F., Reininger, B., Vincent, M. L., Parra-Medina, D., ... \& Taylor, D. (2004). An exploration of the relationship between youth assets and engagement in risky sexual behaviors. Journal of Adolescent Health, 35(5), 424-e21. doi: https://doi.org/10.1016/j.jadohealth.2004.02.008

10. Habib, C., Santoro, J., Kremer, P., Toumbourou, J., Leslie, E., \& Williams, J. (2010). The importance of family management, closeness with father and family structure in early adolescent alcohol use. Addiction, 105(10), 1750-1758. doi: 10.1111/j.13600443.2010.03021.x

11. Heck, K. E., \& Subramaniam, A. (2009). Youth Development Frameworks. [Monograph]. Davis, CA. Recuperado de: http://4h.ucanr.edu/files/1224.pdf

12. Larson, R., \& Richards, M. H. (1994). Divergent realities: The emotional lives of mothers, fathers, and adolescents. New York: BasicBooks.

13. Laursen, B., Coy, K. C., \& Collins, W. A. (1998). Reconsidering changes in parent-child conflict across adolescence: a meta-analysis. 
Child Development, 69(3), 817-832. doi: 10.1111/j.14678624.1998.tb06245.x

14. Leffert, N., Benson, P. L., Scales, P. C., Sharma, A. R., Drake, D. R., \& Blyth, D. A. (1998). Developmental assets: Measurement and prediction of risk behaviors among adolescents. Applied Developmental Science, 2(4), 209-230. doi: http://dx.doi.org/10.1207/s1532480xads0204_4

15. Lerner, R. M., Lerner, J. V., Almerigi, J. B., Theokas, C., Phelps, E., Gestsdottir, S., ... \& Smith, L. M. (2005). Positive youth development, participation in community youth development programs, and community contributions of fifth-grade adolescents: Findings from the first wave of the 4-H study of positive youth development. The Journal of Early Adolescence, 25(1), 17-71. doi: 10.1177/0272431604272461

16. Marshal, M. P., \& Chassin, L. (2000). Peer influence on adolescent alcohol use: The moderating role of parental support and discipline. Applied Developmental Science, 4(2), 80-88. doi: http://dx.doi.org/10.1207/S1532480XADS0402_3

17. Medina-Mora, M.E., Villatoro-Velázquez, J.A., Fleiz-Bautista, C., Téllez-Rojo, M.M., Mendoza-Alvarado, L.R., Romero-Martínez, M., Gutiérrez-Reyes, J.P., ... \& Guisa-Cruz, V. (2012). Encuesta Nacional de Adicciones 2011: Reporte de Alcohol. México: INPRFM. Recuperado de: http://www.conadic.salud.gob.mx/pdfs/ENA_2011_ALCOHOL.pdf

18. Murphey, D. A., Lamonda, K. H., Carney, J. K., \& Duncan, P. (2004). Relationships of a brief measure of youth assets to healthpromoting and risk behaviors. Journal of Adolescent Health, 34(3), 184-191. doi: https://doi.org/10.1016/j.jadohealth.2003.05.004

19. Oman, R. F., Vesely, S. K., \& Aspy, C. B. (2005). Youth assets and sexual risk behavior: the importance of assets for youth residing in one-parent households. Perspectives on Sexual and Reproductive Health, 37(1), 25-31. doi: 10.1363/372505

20. Oman, R. F., Vesely, S., Aspy, C. B., McLeroy, K. R., Rodine, S., \& Marshall, L. (2004). The potential protective effect of youth assets on adolescent alcohol and drug use. American Journal of Public Health, 94(8), 1425-1430. doi: 10.2105/AJPH.94.8.1425

21. Pagan R. M., \& DePaulo, D. (2013). The role of family support and parental monitoring as mediators in Mexican American adolescent drinking. Substance Use \& Misuse, 48(14), 1577-1588. doi: 10.3109/10826084.2013.808220

22. Ryan, S. M., Jorm, A. F., \& Lubman, D. I. (2010). Parenting factors associated with reduced adolescent alcohol use: a systematic review 
of longitudinal studies. Australian \& New Zealand Journal of Psychiatry, 44(9), 774-783. doi: 10.1080/00048674.2010.501759

23. Sánchez-Xicotencatl, C. O. (2015). Factores de protección/riesgo en adolescentes consumidores de alcohol (Tesis de Doctorado). Universidad Nacional Autónoma de México.

24. Scales, P. C. (1999). Reducing risks and building developmental assets: Essential actions for promoting adolescent health. Journal of School Health, 69(3), 113-119. doi: 10.1111/j.17461561.1999.tb07219.x

25. Stavrinides, P., Georgiou, S., \& Demetriou, A. (2010). Longitudinal associations between adolescent alcohol use and parents' sources of knowledge. British Journal of Developmental Psychology, 28(3), 643-655. doi: 10.1348/026151009X466578

26. Steinberg, L., \& Morris, A. S. (2001). Adolescent development. Journal of Cognitive Education and Psychology, 2(1), 55-87. doi: https://doi.org/10.1146/annurev.psych.52.1.83

27. Tebes, J.K., Cook, E. C., Vanderploeg, J. J., Feinn, R., Chinman, M. J., Shepard, J. K., ... \& Connell, C. M. (2011). Parental knowledge and substance use among African American adolescents: Influence of gender and grade level. Journal of Child and Family Studies, 20(4), 406-413. doi: doi:10.1007/s10826-010-9406-3

28. Villatoro, V. J., Medina-Mora, I. M. E., Martín del Campo, S. R., Fregoso, I. D., Bustos, G. M., Resendiz, E. E., ... \& Cañas, M. V. (2016). El consumo de drogas en estudiantes de México: tendencias y magnitud del problema. Salud Mental, 39(4), 193-203. 